\title{
Elastic anomalies in glasses: Elastic string theory understanding of the cases of glycerol and silica
}

\author{
Ernesto Bianchi $\odot,{ }^{1,2}$ Valentina M. Giordano $\odot,{ }^{3}$ and Fernando Lund $\oplus^{1,4}$ \\ ${ }^{1}$ Departamento de Física, Facultad de Ciencias Físicas y Matemáticas, Universidad de Chile, Santiago, Chile \\ ${ }^{2}$ Instituto de Física, Pontificia Universidad Católica de Chile, Casilla 306, Santiago, Chile \\ ${ }^{3}$ Institut Lumière Matière, UMR 5306 Université Lyon 1-CNRS, F-69622 Villeurbanne Cedex, France \\ ${ }^{4}$ CIMAT, Facultad de Ciencias Físicas y Matemáticas, Universidad de Chile, Santiago, Chile
}

(Received 31 January 2020; revised manuscript received 26 March 2020; accepted 1 May 2020; published 22 May 2020)

\begin{abstract}
In this work we present an implementation of the analytical string theory recently applied to the description of glasses. These are modeled as continuum media with embedded elastic string heterogeneities, randomly located and randomly oriented, which oscillate around a straight equilibrium position with a fundamental frequency depending on their length. The existence of a length distribution is reflected then in a distribution of oscillation frequencies which is responsible for the boson peak in the glass density of states. Previously, it has been shown that such a description can account for the elastic anomalies reported at frequencies comparable with the boson peak: the strong phonon scattering and the negative dispersion in the sound velocity, as a result of the interference of the string oscillations with propagating sound plane waves. Here we start from the generalized hydrodynamics to determine the dynamic correlation function $S(k, \omega)$ associated with the coherent, dispersive, and attenuated sound waves resulting from such interference. We show that once the vibrational density of states has been measured, we can use it for unambiguously fixing the string length distribution inherent to a given glass. The density-density correlation function obtained using such distribution is strongly constrained, and able to account for the experimental data collected on two prototypical glasses with very different microscopic structure and fragility: glycerol and silica. The obtained string length distribution is compatible with the typical size of elastic heterogeneities previously reported for silica and supercooled liquids, and the atomic motion associated with the string dynamics is consistent with the soft modes recently identified in large-scale numerical simulations as nonphonon modes responsible for the boson peak. The theory is thus in agreement with the most recent advances in the understanding of the glass-specific dynamics and offers an appealing, simple understanding of the microscopic origin of the latter, while raising new questions on the universality or material specificity of the string distribution properties.
\end{abstract}

DOI: 10.1103/PhysRevB.101.174311

\section{INTRODUCTION}

A long-standing issue in condensed matter physics is represented by the understanding of the low-frequency vibrational properties of glasses. It is well known that at long wavelengths and low frequencies, the continuum description of elastic properties in a solid generally works well in a crystal, which means that the Debye approximation of the density of states is a good description of the actual situation [1]. This approximation however dramatically fails when it comes to a disordered material such as a glass, in spite of intuition that says this should not happen. Indeed, on the macroscopic scale, the wavelengths of sound waves are much larger than the typical length scale of the disorder; thus the microscopic structure should be irrelevant, and a description of the medium as a continuum should work well. We can expect this approximation to fail when the wavelength becomes comparable with the microscopic structure. It is however found to fail much earlier than expected, at wavelengths on the order of tens of mean interatomic distances, a mesoscopic scale corresponding to the medium-range order in glasses and at which it still works reasonably well in crystals [2].

Historically, the above failure has been observed through the deviation of the vibrational density of states from the
Debye prediction at frequencies of a few $\mathrm{THz}$, about $1 / 10$ of the Debye frequency, pointing to the existence of an excess of low-energy modes, called the "boson peak" [3]. Such anomaly in the vibrational density of states can be connected with the anomalous temperature dependence of the specific heat at low temperature, as well as with the existence of a plateau in the thermal conductivity, with the suppression of the umklapp peak typical of crystals, at temperatures of about $10 \mathrm{~K}[4,5]$. Such excess is qualitatively similar for many such materials, and the details, but not the broad features, depend on external parameters such as temperature, density, pressure, as well as chemical and thermal history [6-15].

This anomaly in the density of states is accompanied by some so-called elastic anomalies in the longitudinal acoustic phonons, as evidenced by a series of experimental studies [2,16-18]: a negative dispersion of the sound velocity, with a minimum close to the boson peak frequency, and a strong attenuation $\Gamma$ of the acoustic phonons, whose dependence on the wave vector $k$ has long been believed to be compatible with a Rayleigh scattering mechanism $\left(\Gamma \propto k^{d+1}\right.$, where $d$ is the dimensionality of space), but recently found to go like $-k^{d+1} \ln k$ near the boson peak frequency [19,20]. As such, the failure of the Debye approximation seems to be 
drawn by a strong scattering of sound waves, whose origin is still a matter of debate and which leads to a crossover from well-defined acoustic modes for long wavelengths to ill-defined ones for smaller wavelengths, the so-called IoffeRegel crossover. Three dynamical regimes have thus been identified in glasses [21,22]: (i) a low-frequency plane-wavedominated regime, where phonons undergo weak scattering and are equivalent to phonons in crystals; (ii) a strongscattering regime, above the Ioffe-Regel limit, where the corresponding phonons are called "diffusons" and cannot be thought of as propagative plane waves anymore [23]; and (iii) the Anderson localization regime near the mobility edge $[21,24,25]$. The existence of these three regimes strongly impacts transport properties, so that their microscopic understanding has become essential for many technological applications aiming at using glasses for thermal insulation or confinement.

Understanding the microscopic origin of the strongscattering regime, and thus of elastic anomalies and the boson peak, represents a challenge that has been at the focus of an intense theoretical and experimental research effort for at least 50 years. Many theories and models have been developed [26-33], which can be divided into three main groups: (i) the existence of quasilocal vibrational states, produced by soft anharmonic potentials [34-36], (ii) the existence of nanometric elastic heterogeneities [27-30,37,38], and (iii) the identification of the boson peak with the first transverse Van Hove singularity, broadened by disorder and shifted because of the density difference with respect to the corresponding crystalline phase $[39,40]$.

Recent work has shown that the theory of elastic nanoheterogeneities is unable to reproduce the observed overRayleigh acoustic attenuation [20]. On the other hand, recent large-scale numerical simulations have unveiled the presence of low-energy quasilocalized nonphononic modes, which would coexist with normal acoustic phonons [41-45] and be characterized by a particle displacement decreasing like $r^{-2}$ in $3 \mathrm{D}$, where $r$ is the distance away from the center of the mode. The low-frequency density of states is thus the result of the sum of a Debye contribution for the acoustic phonons and a nonphononic contribution coming from these modes and going as $\omega^{4}$. These low-energy modes would stem out from microscopic structural arrangements and/or heterogeneities in the glass and can be associated with another class of theoretical models, which has focused on the identification of stringlike behavior in glasses. Cooperative stringlike motion was successively found in supercooled liquids [46,47], and Yu et al. [48] proposed an interpretation of the $\beta$ relaxation in a variety of supercooled liquids and glasses in terms of stringlike configurations. Concerning glasses, already in the 1990s Schober et al. [49] in a numerical simulation of a soft-sphere glass identified well below the glass transition temperature vibrational modes involving only atoms arranged in a stringlike pattern. Novikov and Surotsev [50] showed that Raman scattering data from glasses could indeed be explained by vibrational eigenmodes localized along a one-dimensional spatial geometry. More recently, Concustell et al. [51] have induced and characterized elastic anisotropy in a bulk metallic glass, showing that their findings are consistent with an alignment of stringlike atomic arrangements.
Recently, one of us has developed an analytical description showing that the presence of strings in a glass thought of as a continuum medium can give rise to the vibrational anomalies observed in glasses [52]. Here we develop this theory and provide a formula to directly link the density of states $g(\omega)$ of an amorphous material in the $\mathrm{THz}$ range with its longitudinal dynamic structure factor $S(k, \omega)$. We find that the string distribution obtained from the experimental boson peak is able to nicely fit the inelastic x-ray scattering data on the $S(q, \omega)$ obtained on glycerol [2] and silica [16] with an agreement as good as the one historically obtained fitting the same data with a damped harmonic oscillator (DHO) model.

This paper is organized as follows: Section II shows how to compute the dynamic structure factor $S(\vec{k}, \omega)$ for coherent acoustic waves propagating in a medium described by a complex index of refraction, starting from the boson peak. This computation is done following our previous work [52] and relies on the properties of an acoustic wave propagating in a medium filled with elastic strings randomly placed and oriented [52,53]. For the reader's convenience, some details of the index of refraction computation are reproduced in Appendix A. The relation of the current approach to the damped harmonic oscillator model is discussed in Appendix B. Section III is devoted to the application of the model for fitting experimental data on two prototypical glasses, glycerol and silica. The discussion of the results of such fitting, and notably the obtained string length distribution and its relation to the elastic anomalies, is reported in Sec. IV A, while Sec. IV B focuses on the comparison of our results and recent numerical simulations, specifically concerning the atomic motion in the strings implicit to the our model. Finally, Sec. V presents our concluding remarks.

\section{THE STRING MODEL}

Here we recall the basics of the model developed in [52,53]. It is based on continuum mechanics. The glass is modeled as a Debye solid plus an embedded distribution of elastic strings. These strings are pinned at their ends and have a distribution of lengths. They can oscillate around an equilibrium position and their fundamental mode of vibration gives rise to the excess modes over the Debye level observed in many glasses. Also, they interact with elastic waves, leading to the frequency dependence of phase velocity and attenuation of coherent sound waves.

\section{A. Preliminaries}

We consider then an elastic, homogeneous, isotropic solid with Lamé coefficients $\lambda, \mu$, mass density $\rho$, and number density $n$. The displacement at time $t$ of a point whose equilibrium position is $\vec{x}$ is denoted $\vec{u}(\vec{x}, t)$, and its velocity is $\vec{v}(\vec{x}, t) \equiv \partial \vec{u}(\vec{x}, t) / \partial t$. The current $\vec{j} \equiv n \vec{v}$ has a longitudinal component $j(\vec{x}, t)$ that, in the absence of strings, obeys the equation

$$
-\frac{1}{c_{L}^{2}} \frac{\partial^{2} j(\vec{x}, t)}{\partial t^{2}}+\nabla^{2} j(\vec{x}, t)+\xi \nabla^{2} \frac{\partial j(\vec{x}, t)}{\partial t}=0,
$$

where $c_{L}^{2}=\rho /(\lambda+2 \mu)$ and $\xi$ is a phenomenological damping coefficient [54]. Looking for traveling waves along one 
dimension,

$$
j(\vec{x}, t)=j_{0} e^{i(\omega t-k x)},
$$

leads to the dispersion relation,

$$
\frac{\omega^{2}}{c_{L}^{2}}-k^{2}-i k^{2} \omega \xi=0,
$$

and, when $\omega \xi \ll 1$, we have

$$
j(\vec{x}, t)=j_{0} e^{i\left(\omega t-\omega x / c_{L}\right)} e^{-x\left(\omega^{2} \xi\right) / 2 c_{L}},
$$

the well-known result that, at low frequencies, elastic waves have a damping proportional to frequency squared, or that there is an attenuation length proportional to $\omega^{2}$. For later purposes we introduce the ("bare") Green's function $g_{0}\left(\vec{x}, \vec{x}_{0}, t-\right.$ $\left.t_{0}\right)$, the solution of

$$
\begin{aligned}
& -\frac{1}{c_{L}^{2}} \ddot{g}_{0}\left(\vec{x}, \vec{x}_{0}, t-t_{0}\right)+\nabla^{2} g_{0}\left(\vec{x}, \vec{x}_{0}, t-t_{0}\right) \\
& \quad+\xi \nabla^{2} \dot{g}_{0}\left(\vec{x}, \vec{x}_{0}, t-t_{0}\right)=-\delta\left(\vec{x}-\vec{x}_{0}\right) \delta\left(t-t_{0}\right),
\end{aligned}
$$

where an overdot denotes differentiation with respect to time. We shall use the definition of the Fourier transform $\tilde{F}(\vec{k}, \omega)$ of a function $F(\vec{x}, t)$ as

$$
\tilde{F}(\vec{k}, \omega) \equiv \int_{-\infty}^{\infty} d^{3} x d t e^{i(\vec{k} \cdot \vec{x}-\omega t)} F(\vec{x}, t),
$$

so the bare Green's function in frequency-wave-number space is given by

$$
\tilde{g}_{0}(\vec{k}, \omega)=\frac{-1}{\left(\omega^{2} / c_{L}^{2}\right)-k^{2}-i k^{2} \xi \omega},
$$

whose poles reproduce the dispersion relation (3).

If now we consider the solid to be in thermodynamic equilibrium at temperature $T$, the currents will be fluctuating quantities. Of particular interest is the dynamic correlation

$$
J(\vec{k}, t) \equiv\left\langle j^{*}(\vec{k}, 0) j(\vec{k}, t)\right\rangle,
$$

where the brackets denote a thermal average. Also, we shall drop the tildes from the Fourier transforms to simplify the notation. Note that because of thermal equilibrium, this is a real function that is defined for positive times $t$, and it can be extended to negative times imposing that it be even in time. Of particular interest is the Fourier transform

$$
\begin{gathered}
J(\vec{k}, \omega) \equiv 2 \operatorname{Re}[\mathcal{J}], \\
\mathcal{J} \equiv \int_{0}^{\infty} d t e^{-i \omega t} J(\vec{k}, t) .
\end{gathered}
$$

A standard treatment [55] of the initial value problem of Eqs. (1) and (5) leads to an expression of the bare correlation $J_{0}$ in terms of the bare Green's function $g_{0}$ :

$$
\begin{aligned}
J_{0}(\vec{k}, \omega) & =-2 \frac{J_{0}(\vec{k}, 0)}{c_{L}^{2}} \omega \operatorname{Im}\left[g_{0}(\vec{k}, \omega)\right] \\
& =\frac{2}{c_{L}^{2}} J_{0}(k, 0) \frac{\xi \omega^{2} k^{2}}{\left(\frac{\omega^{2}}{c_{L}^{2}}-k^{2}\right)^{2}+\xi^{2} \omega^{2} k^{4}},
\end{aligned}
$$

where the second line is a well-known expression [56], that has been obtained here substituting (7) into (11).

\section{B. Addition of strings}

We now consider elastic waves as above, propagating in a medium with a frequency-dependent index of refraction induced by the presence of strings:

$$
\begin{aligned}
& -\frac{1}{c_{L}^{2}} \frac{\partial^{2} j(\vec{x}, t)}{\partial t^{2}}+\nabla^{2} j(\vec{x}, t)+\xi \nabla^{2} \frac{\partial j(\vec{x}, t)}{\partial t} \\
& -\nabla^{2}[f(t) * j(\vec{x}, t)]=0,
\end{aligned}
$$

where $*$ means convolution in time and $f(t)$ must vanish for negative times due to causality. The road to Eq. (13) as well as its relation to strings is recalled in Appendix A. There is a Green's function associated with this equation, the solution to

$$
\begin{aligned}
-\frac{1}{c_{L}^{2}} & \frac{\partial^{2} g\left(\vec{x}, \overrightarrow{x_{0}}, t-t_{0}\right)}{\partial t^{2}}+\nabla^{2} g\left(\vec{x}, \overrightarrow{x_{0}}, t-t_{0}\right) \\
& \quad+\xi \nabla^{2} \frac{\partial g\left(\vec{x}, \overrightarrow{x_{0}}, t-t_{0}\right)}{\partial t}-\nabla^{2}\left[f(t) * g\left(\vec{x}-\overrightarrow{x_{0}}, t-t_{0}\right)\right] \\
= & -\delta\left(\vec{x}-\overrightarrow{x_{0}}\right) \delta\left(t-t_{0}\right),
\end{aligned}
$$

which gives, in frequency-wave-number space,

$$
g(\vec{k}, \omega)=\frac{-1}{\left(\omega^{2} / c_{L}^{2}\right)-k^{2}-i k^{2} \xi \omega+k^{2} f(\omega)},
$$

whose poles give an effective, complex wave number as a function of frequency, say $K(\omega)$, that defines a phase velocity, $v \equiv \omega / \operatorname{Re}[K(\omega)]$, and an attenuation length, $\ell \equiv$ $-1 / \operatorname{Im}[K(\omega)]$. Alternatively, both the phase velocity and the attenuation length can be expressed as functions of the wave number $k$.

Consider now Eq. (13) in wave number space:

$$
\begin{gathered}
-\frac{1}{c_{L}^{2}} \frac{\partial^{2} j(\vec{k}, t)}{\partial t^{2}}-k^{2} j(\vec{k}, t)-k^{2} \xi \frac{\partial j(\vec{k}, t)}{\partial t} \\
+k^{2} \int_{-\infty}^{\infty} d t^{\prime} f\left(t^{\prime}\right) j\left(\vec{k}, t-t^{\prime}\right)=0 .
\end{gathered}
$$

Multiplying this equation by $j^{*}(\vec{k}, 0)$, and taking the thermal average, we get

$$
\begin{aligned}
& \frac{-1}{c_{L}^{2}} \frac{\partial^{2} J(\vec{k}, t)}{\partial t^{2}}-k^{2} J(\vec{k}, t)-k^{2} \xi \frac{\partial J(\vec{k}, t)}{\partial t} \\
& \quad+k^{2} \int_{-\infty}^{t} d t^{\prime} f\left(t^{\prime}\right) J\left(\vec{k}, t-t^{\prime}\right)+k^{2}\left\langle j^{*}(\vec{k}, 0) \mathcal{I}(\vec{k}, t)\right\rangle=0 .
\end{aligned}
$$

This equation is similar to, but differs from, the equation obeyed by the dynamic form factor in a memory function formalism [57]. This point is further discussed in Appendix B. Further multiplying by $\exp (-i \omega t)$ and integrating over $t$ from 0 to $\infty$ leads to

$$
\begin{aligned}
& \frac{1}{c_{L}^{2}} \frac{\partial J(\vec{k}, 0)}{\partial t}+k^{2} \xi J(\vec{k}, 0)+k^{2} D(\vec{k}, \omega)+i \frac{J(\vec{k}, 0)}{c_{L}^{2}} \omega \\
& \quad-g^{-1}(\vec{k}, \omega) \mathcal{J}(\vec{k}, \omega)=0,
\end{aligned}
$$

where

$$
D(\vec{k}, \omega) \equiv \int_{0}^{\infty} d t e^{-i \omega t}\left\langle j^{*}(\vec{k}, 0) \mathcal{I}(\vec{k}, t)\right\rangle,
$$


and

$$
\mathcal{I}(\vec{k}, t) \equiv \int_{0}^{\infty} d \tau f(\tau+t) j(\vec{k},-\tau)
$$

is a functional of the history of the system previous to its initial state $j(\vec{k}, 0)$. The initial value problem for dispersive waves, i.e., when the speed of propagation depends on frequency, does in general depend on its previous history. We shall assume there is no statistical correlation between the initial value of the current $j^{*}(\vec{k}, 0)$ and its previous, dispersiondominated, history:

$$
D(\vec{k}, \omega)=0
$$

We now show that

$$
\frac{1}{c_{L}^{2}} \frac{\partial J(\vec{k}, 0)}{\partial t}+k^{2} \xi J(\vec{k}, 0)=0 .
$$

Indeed, note that Eq. (17) is the time derivative of

$$
\begin{gathered}
-\frac{1}{c_{L}^{2}} \frac{\partial j(\vec{k}, t)}{\partial t}-k^{2} j(\vec{k}, t)-n k^{2} \xi u(\vec{k}, t) \\
+n k^{2} \int_{-\infty}^{\infty} d t^{\prime} f\left(t^{\prime}\right) u\left(\vec{k}, t-t^{\prime}\right)=0 .
\end{gathered}
$$

Since $\left\langle J_{l \vec{k}}^{*}(0) u_{l \vec{k}}(0)\right\rangle=0$ because density and velocity are uncorrelated, and assuming again that the initial value of the current is uncorrelated with the previous, dispersion-dominated, history, we have (22). Consequently, Eqs. (18) and (9) lead to

$$
J(\vec{k}, \omega)=-\frac{2}{c_{L}^{2}} J(k, 0) \operatorname{Im}[\omega g(\vec{k}, \omega)],
$$

where $g(\vec{k}, \omega)$ is the Green's function and which leads to the density correlation through

$$
S(\vec{k}, \omega)=\frac{k^{2}}{\omega^{2}} J(\vec{k}, \omega) .
$$

\section{Relation of the boson peak to the strings}

Following [52], as we explain in more detail in Appendix A, our model corresponds to a harmonic system with two kinds of normal modes, noninteracting at leading order: phonons and string modes. As such, the normal mode density of states is

$$
g(\omega)=g_{D}(\omega)+g_{S}(\omega),
$$

where $g_{D}(\omega)$ is the Debye distribution, only due to phonons, and $g_{S}(\omega)$ is the excess over this distribution (i.e., the boson peak), assumed to only correspond to string modes. The interaction between phonons and strings is given by the (nonvanishing) right-hand sides of Eqs. (A1) and (A2). This interaction is responsible for the modification of velocity and damping of phonons in the energy range of the boson peak, as we will see in the next sections.

The distribution of excess modes over the Debye distribution determines then a distribution of string lengths $L$ through

$$
g_{S}\left(\omega_{0}\right) d \omega_{0} \equiv 2 p(L) d L
$$

with $\omega_{0}$ the fundamental frequency of a string of length $L$ pinned at its ends. The factor of two in Eq. (27) indicates that there are twice as many states of a given frequency as strings. This is due to the fact that each string can oscillate along two linearly independent directions that are perpendicular to its equilibrium orientation.

As explained in detail in Appendix A, the string has a mass per unit length $m=\alpha \rho b^{2}$, where $\rho$ is the mass density of the material, $b$ is a length of order one interatomic spacing, and $\alpha$ is a dimensionless parameter of order one. The string also has a line tension $\mathcal{T}$ and an internal damping $B / 2 \mathrm{~m}$. Consequently, $c=\sqrt{\mathcal{T} / m}$ is the speed of waves along the string, and $\omega_{0}=\sqrt{(\pi c / L)^{2}-(B / 2 m)^{2}}$. So, given a specific boson peak, as determined by atomistic modeling or experimental data, $g_{S}\left(\omega_{0}\right)$ can be read off it and Eq. (27) determines the string length distribution $p(L)$, which in turn, through (A14), determines the index of refraction $f(\omega)$ that goes into the Green's function (15) whose imaginary part provides the correlation function $S(\vec{k}, \omega)$ using (24) and (25).

\section{RELATING THE BOSON PEAK DATA TO THE IXS DATA}

In this section we relate the derived $S(\vec{k}, \omega)$ to the experimental data that can be obtained in an inelastic x-ray scattering experiment (IXS), in order to apply our model to the data collected with this technique on two prototype glasses, glycerol and silica.

The experimental inelastic scattering spectrum of a glass is composed of a Brillouin triplet: an elastic line, related to the frozen density fluctuations, and the Stokes and antiStokes inelastic peaks corresponding to the annihilation and excitation of a vibrational excitation. The measured spectrum can be formally described as [58]

$$
I(k, \omega)=A_{k} \hbar \omega \frac{\left[n_{B}(\omega)+1\right]}{k_{B} T} S_{\mathrm{tot}}(k, \omega) .
$$

Here, we have replaced the vector $\vec{k}$ by its modulus, given the isotropy of the system. $A_{k}$ is a normalization factor reflecting the $k$ dependence of the atomic form factors, $n_{B}(\omega)=$ $\left(e^{\hbar \omega / k_{B} T}-1\right)^{-1}$ is the Bose-Einstein factor, and $S(k, \omega)$ is the longitudinal density-density correlation function, which is modeled as the sum of a delta function for the elastic line and an inelastic contribution:

$$
S_{\text {tot }}(\vec{k}, \omega)=f_{k} \delta(\omega)+\left(1-f_{k}\right) S(k, \omega),
$$

where $f_{k}$ accounts for the measured proportion between elastic and inelastic parts of spectra and is related to the nonergodicity factor and $S(k, \omega)$ is given by (25).

Equation (28) can be fitted to the experimental spectra after convolution with the instrumental resolution function $R(\omega)$, adding a $k$-dependent background $b g_{k}$ :

$$
I_{\exp }(k, \omega)=b g_{k}+\int I(k, \omega) R\left(\omega-\omega^{\prime}\right) d \omega^{\prime} .
$$

Now that we have a reasonable expression for the intensity to work with, the next task is to assign physical values to the parameters of the model. For the instrumental resolution function, we use the one experimentally measured and we model it via a linear combination of a Lorentzian and a 
Gaussian function. We are then left with three groups of parameters for the description of the experimental spectra:

(i) Experimental setup and partly material-related: $b g_{k}, A_{k}$.

(ii) Material-related: $f_{k}, \xi, J(\vec{k}, 0), c_{L}$, and $c_{T}$.

(iii) Strings-related: $\alpha, c, B / m$, and $g_{S}(\omega)$.

The function $g_{S}(\omega)$ is obtained from the measured density of states, by modeling the excess over Debye distribution using a 5-degree polynomial (i.e., up to $\omega^{5}$ ). Note that the theoretical $g(\omega)$ is a number of states per unit frequency per unit volume. The experimental density of states is, however, normalized to the atomic volume, and thus is a number of states per unit frequency. The extra volume is obtained from the very low frequency asymptotics of the experimental values, where it does coincide with the Debye theory. The number of free parameters can be reduced by relating the velocities $c_{L}, c_{T}$, and $c$ through the equalities: $c_{T}=c_{L} / \sqrt{K / \mu+4 / 3}$, where $K$ and $\mu$ are the material's bulk and shear modulus respectively, and [59] $c=c_{T}$. The zero-frequency value $J(k, 0)$ is fixed to the literature data of the longitudinal velocity as measured with Brillouin light scattering. To simplify our model, we observe that the effect of damping in the elastic waves due to the internal friction of strings, given by $B / m$, can be reasonably assumed to be negligible compared to the other sources of damping. We thus take its value simply as zero.

The remaining fitting parameters are thus $b g_{k}, A_{k}$ for the instrumental part; $f_{k}, \xi$, and $c_{L}$ for the material part; and only $\alpha$ for the strings-related part. $\xi, c_{L}$, and $\alpha$ are expected, in a first approximation, to be $k$ independent. For this reason, and for getting the best description over a large $k$ range, they are found by simultaneously fitting 5 IXS spectra throughout the studied wave number range. The $k$-dependent parameters, such as $b g_{k}, A_{k}$, and $f_{k}$, are then found by independently fitting each spectrum using Eq. (30), for fixed values of $\xi, c_{L}$, and $\alpha$. The procedure is iteratively repeated until convergence.

To sum up, given boson peak data $g_{S}(\omega)$, one obtains $p(L)$ through Eq. (27). This relation involves a free parameter $B / 2 m$, the internal damping of string motion, that in the examples that will be worked out below will be put equal to zero. It also involves $c$, the speed of waves along the string, a parameter that is completely determined by $c_{T}$, the transverse speed of sound of the material under consideration. Next, $p(L)$ determines $f(\omega)$ through Eq. (A13). This relation involves $m$, the mass per unit length of the string, which is related to the mass density $\rho$ of the material through $m=\alpha \rho b^{2}$, where $b$ is a length of order one interatomic distance that cancels out in the calculations, and $\alpha$ is a dimensionless parameter of order one that, in the examples below, will be effectively put equal to one. Finally, as mentioned, $J(k, 0)$ is also fixed from literature data. With $f(\omega)$ determined, $S(\vec{k}, \omega)$ is found using Eqs. (15), (24), and (25). This final formula for $S(\vec{k}, \omega)$ depends on two parameters: $\xi$, the wave attenuation coefficient in the absence of strings, and $c_{L}$, the speed of sound. They will be varied, within reasonable bounds, to fit the $S(k, \omega)$ obtained from the experimental $I(k, \omega)$. This, in turn, is done using Eqs. (28) and (29). These expressions depend on experimental parameters, $A_{k}$ and $b g_{k}$, and a material-related parameter, $f_{k}$.

Once the parameters that afford the best fit to the experimental data are obtained, they can be used to obtain the phase

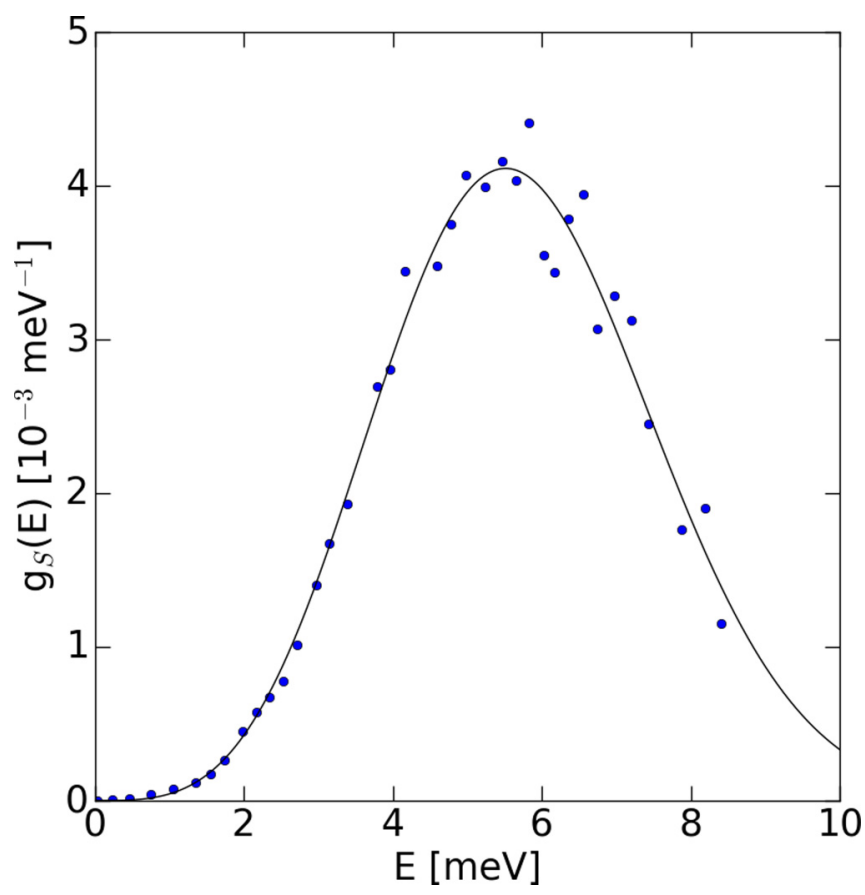

FIG. 1. The boson peak of glassy glycerol at $170 \mathrm{~K}$, taken from Ref. [60], and reported in nonreduced units $\left[g(\omega)-g_{D}(\omega)\right]$, together with the fit to the function defined in the text.

velocity $v$ and attenuation $\Gamma$ solving for the poles, say $K(\omega)$, of the Green's function in Eq. (15):

$$
\begin{gathered}
v=\frac{\omega}{\operatorname{Re}[K(\omega)]}, \\
\Gamma=-c_{L} \operatorname{Im}[K(\omega)] .
\end{gathered}
$$

For testing the modeling of the inelastic scattering data through the string distribution obtained from the experimental density of states, we have chosen two prototype cases, a fragile glass, glycerol, and a strong one, silica, for which all the experimental data are available.

\section{A. The case of glycerol}

We present here the fit of inelastic $\mathrm{x}$ ray scattering data obtained by Monaco and Giordano [2] on a glass of glycerol at $150 \mathrm{~K}$, the glass transition temperature being $T_{g}=189 \mathrm{~K}$. In that paper, data between $k=1.1$ and $k=5 \mathrm{~nm}^{-1}$ have been reported and fitted with a damped harmonic oscillator (DHO) model for the inelastic contribution. Such work has given the first experimental evidence of the existence of elastic anomalies in glasses in the boson peak region and the early breakdown of the Debye approximation: a crossover from a regime where the phonon attenuation increases with $k^{4}$ to a regime where it goes as $k^{2}$, accompanied by a dip in the sound velocity with respect to the expected behavior. Moreover, the calculation of the density of states using the experimental $S(k, \omega)$ has been found to be in reasonable agreement with the experimental data as measured by inelastic neutron scattering at $170 \mathrm{~K}$ [60]. The role of the attenuation has been there highlighted, as fundamental to get the right position of the boson peak. 


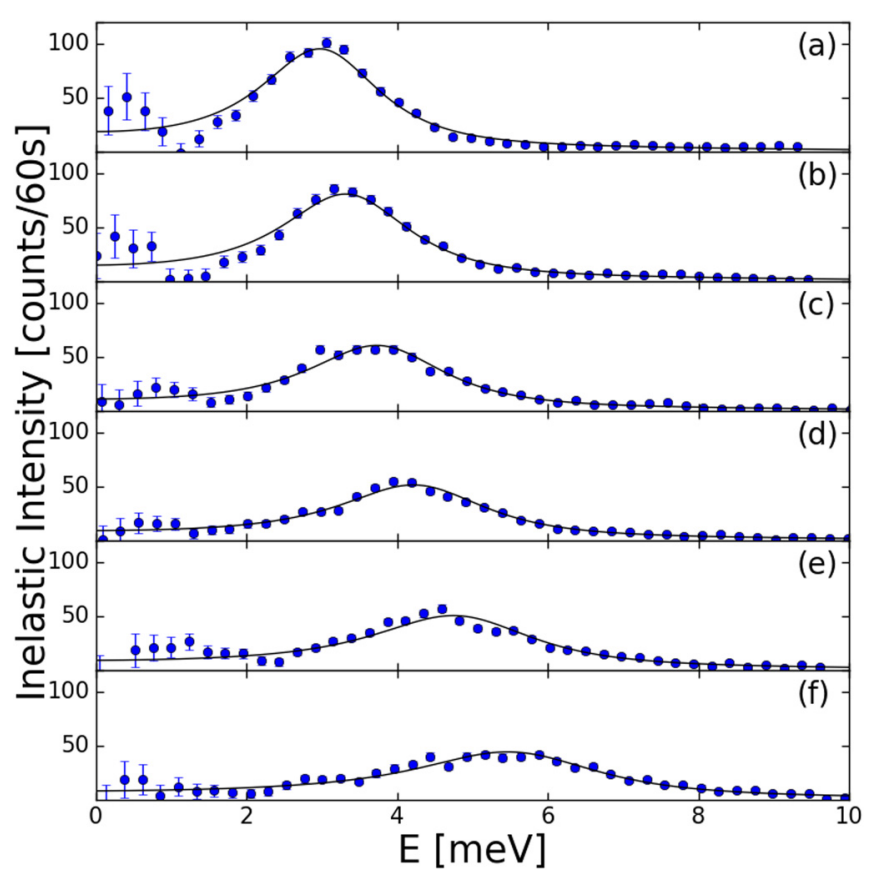

FIG. 2. Inelastic x-ray scattering spectra of glassy glycerol as measured at $150 \mathrm{~K}$ and reported in Ref. [2] for exchanged momentum $q=1.3,1.5,1.7,1.9,2.1$, and $2.5 \mathrm{~nm}^{-1}$ from panels (a) to (f). For the sake of clarity we report only the positive energy exchange side and only the inelastic contribution after subtraction of the elastic line. The black solid lines represent the fit with the string model, where the string distribution has been deduced from the boson peak.

If in that work the authors could reproduce the density of states from the experimental density-density correlation function, here we follow the opposite direction, using the density of states for reproducing the $S(k, \omega)$. Figure 1 reports the excess density of states on the Debye prediction as obtained from Ref. [60]. In order to extract an analytical expression for $p(L)$, we fit it using the function $g_{s}(E)=$ $p 0 * E^{3} * \exp \left[-p 1 *(E-p 2)^{2}\right]$, which respects the boundary conditions $g_{s}(0)=g_{s}(\infty)=0$, also reported in the figure. Figure 2 reports a selection of inelastic spectra of glycerol, in the $[1-3] \mathrm{nm}^{-1}$ range, together with the fit to Eq. (28). For a better visualization, we have chosen to report only the inelastic part of the spectra, after subtraction of the fitted elastic line, and only for positive exchanged energies.

We recall here that in Ref. [2] the $k^{4}-k^{2}$ crossover was identified at $k \approx 2 \mathrm{~nm}^{-1}$. The $k$ values here chosen span then the interesting $k$ region. We have not reported larger $k$ as the vibrational modes are largely too damped and the energy range too short for a constraining fit with our model. From the figure, it is clear that the fitting model catches quite well the $k$ dependence of the major inelastic contribution features (energy position and attenuation), with a fitting quality comparable to the DHO fit reported in Ref. [2]. The $k$-independent parameters fitted with our iterative procedure are reported in Table I.

To go further, we can extract from our model the phase velocity $v$ and the attenuation $\Gamma$ calculated as indicated in Eqs. (31) and (32). They are reported in Fig. 3, together with the boson peak, and they are compared with the expected
TABLE I. Table of the physical quantities needed for the string model and the $k$-independent fitting parameters for glycerol and silica.

\begin{tabular}{lcc}
\hline \hline & Glycerol & Silica \\
\hline$J(k, 0)(\mathrm{km} / \mathrm{s})$ & $3.625[61]$ & $6.5[16]$ \\
$K(\mathrm{GPa})$ & $14.1917[62,63]$ & $44.97[64]$ \\
$\mu(\mathrm{GPa})$ & $4.85[62,63]$ & $36.07[64]$ \\
$c_{L}(\mathrm{~km} / \mathrm{s})$ & $3.42 \pm 0.03$ & $6.08 \pm 0.05$ \\
$\xi\left(10^{-14} \mathrm{~s}\right)$ & $3.1 \pm 0.3$ & $2.84 \pm 0.17$ \\
\hline \hline
\end{tabular}

velocity and attenuation in the absence of strings (in red). It is clear that the addition of strings strongly modifies velocity and attenuation. More precisely, from these graphs, we understand that the longest strings, corresponding to the low-energy modes of the boson peak (BP), and thus to its onset, interfere with elastic waves slowing them down, while the shortest strings, corresponding to the high-energy part of the BP, speed them up again. This interference is accompanied by the onset of a strong attenuation. Note that in the figure we report the "string-dependent" attenuation, i.e., the residual attenuation once the one without strings has been subtracted. As such, at

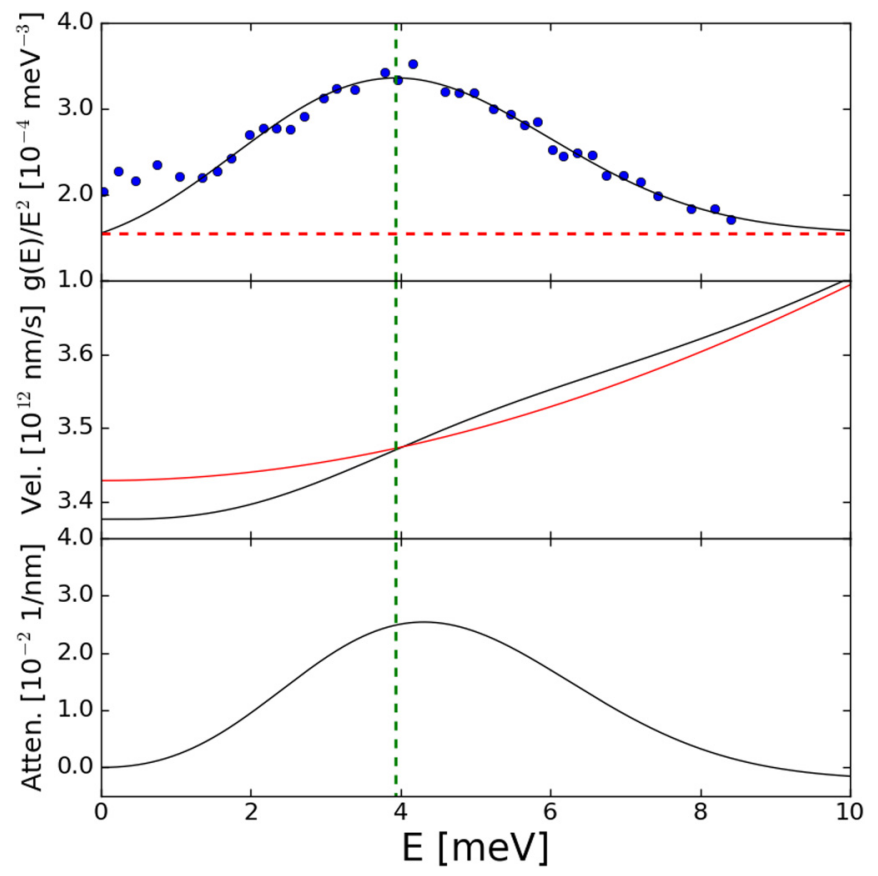

FIG. 3. Top panel: Excess of modes (boson peak) in the regular density of states of glassy glycerol and its modeling with the function detailed in the text. The red dashed line represents what we should have in the absence of strings (no excess). The mismatch at low energy is due to the larger weight that low-energy states have in this case, compared with the fit in nonreduced units reported in Fig. 1. Central panel: The velocity as obtained using Eq. (31), compared with the one in the case of no strings (red). Bottom panel: The excess attenuation, due to strings, with respect to the attenuation in the case of no strings. Its maximum, marked by the vertical green dashed line, corresponds to the change of behavior in the velocity from a lower to a higher velocity with respect to the no-string case, and to the maximum of the $\mathrm{BP}$. 


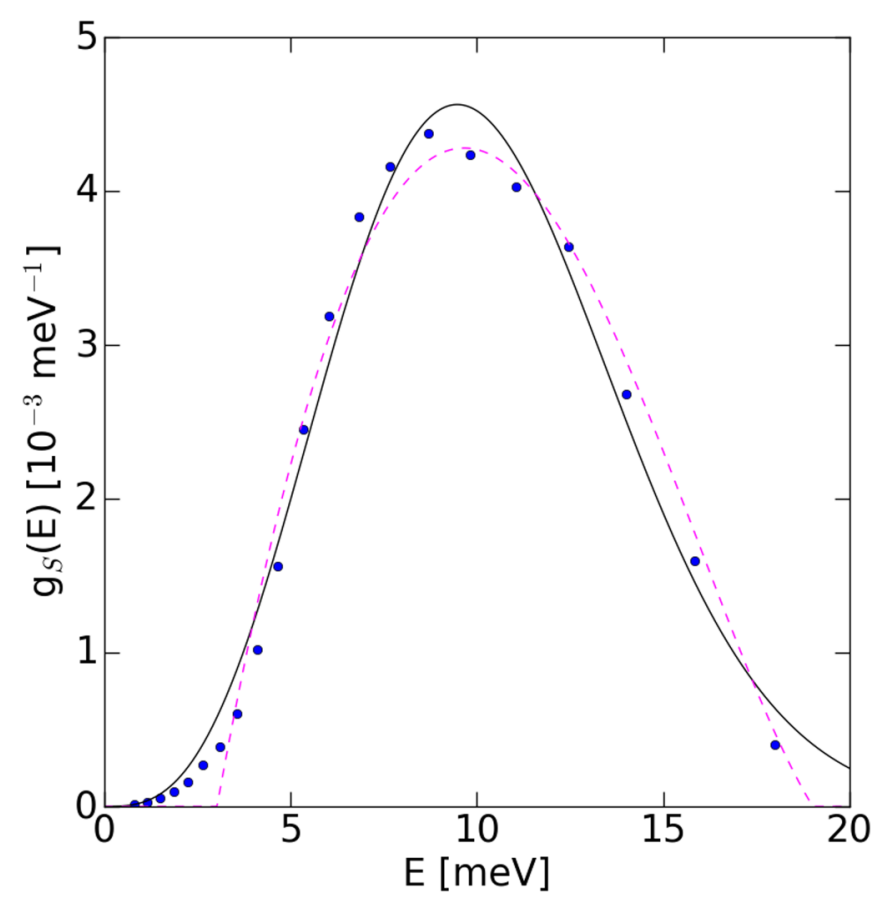

FIG. 4. The boson peak of glassy silica at $1673 \mathrm{~K}$, taken from Ref. [17], reported in nonreduced units $\left[g(\omega)-g_{D}(\omega)\right]$, together with the fit to the function detailed in the text, and a fit to a 5-degree polynomial for comparison.

low energy it is zero and constant, meaning that the total attenuation goes like $k^{2}$, and then it increases, meaning that the total one goes like $k^{4}$, before decreasing again. The maximum corresponds to the maximum of the BP, i.e., the maximum of density of string modes. It is worth noticing that while our model well reproduces the existence of a dip in the sound velocity as well as the $k^{4}-k^{2}$ crossover, all the phenomenology appears shifted at lower $k$ with respect to what is found with a DHO model. We do not expect the two models to give exactly the same results, as we explain in Appendix B; still the physical meaning behind the results obtained with the two models is very similar: we have the same elastic anomalies, which occur in both cases in correspondence with the BP, excess of modes in the regular density of states (string case) or the reduced one (divided by $\omega^{2}$; DHO case).

\section{B. The case of silica}

In this section, we apply the string model to the case of silica and more specifically we fit the data collected by $\mathrm{x}$-ray inelastic scattering at $T=1620 \mathrm{~K}$ (glass transition temperature $T_{g}=1450 \mathrm{~K}$ [65]) and reported by Baldi et al. [17]. Figure 4 reports the excess density of states in nonreduced units as obtained from the one measured by neutron scattering at $T=1673 \mathrm{~K}$ and reported in the same paper, together with a fit to the same function introduced for glycerol, for extracting the string distribution. This function overestimating the low-energy density of states in this case, we have fitted the $\mathrm{BP}$ as well with a 5-degree polynomial function, cutting it at low and high energies to avoid unphysical negative values. While the choice of the analytical function for the BP does modify the quality of the fit for it, we have verified that

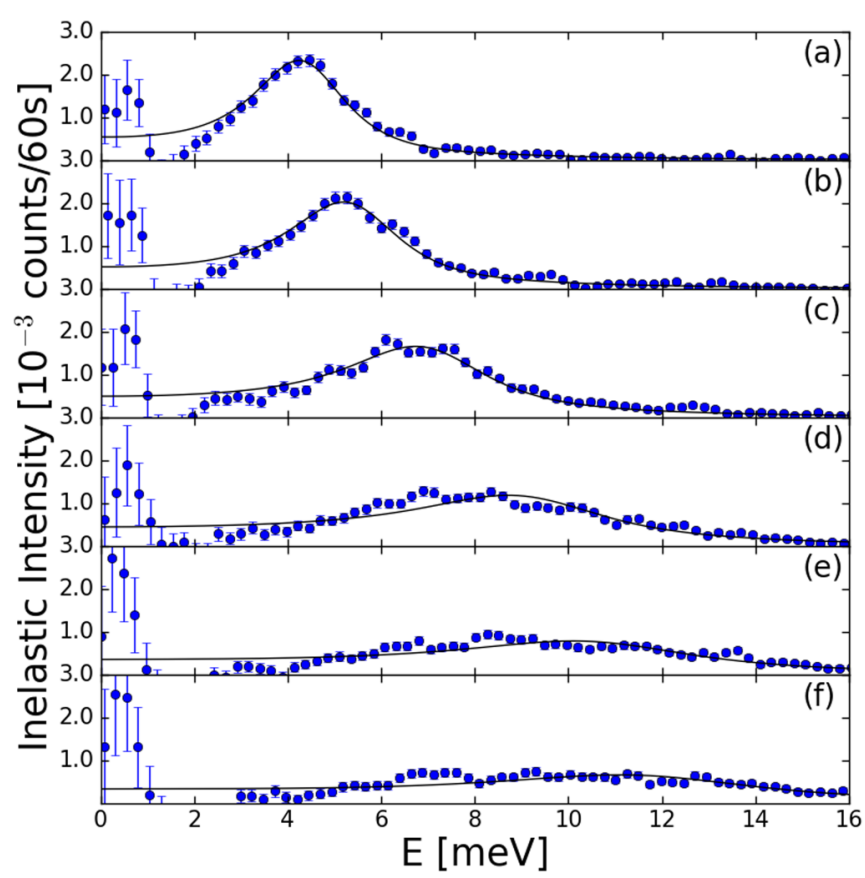

FIG. 5. Inelastic x-ray scattering spectra of glassy silica as measured at $1620 \mathrm{~K}$ and reported in Ref. [17] for exchanged momentum $q=1.1,1.3,1.7,2.3,2.7$, and $2.9 \mathrm{~nm}^{-1}$ from panels (a) to (f). For the sake of clarity we report only the positive energy exchange side and only the inelastic contribution after subtraction of the elastic line. The black lines represent the fit with the string model, where the string distribution has been deduced from the boson peak using the same fitting function as in glycerol.

the string distribution and the inelastic spectra fit are not significantly modified, and we can draw the same general conclusions independently on the specific model chosen. As such, in the following we show only the results obtained with the same fitting function as used for glycerol, which is physically more sound. Some selected inelastic spectra with the fit performed with our model in the exchanged wave vector range between 0.95 and $2.8 \mathrm{~nm}^{-1}$ are reported in Fig. 5. The agreement is quite good, up to $k \sim 2 \mathrm{~nm}^{-1}$, from which value it gets worse and worse with increasing wave vector, overestimating the position and underestimating the width. The $k$-independent fitting parameters are reported in Table I. This can be understood going back to Ref. [17], where a critical wave vector $k_{c}=1.41 \mathrm{~nm}^{-1}$ was identified, such that $\Gamma \propto k^{4}$ for $k \ll k_{c}$ and $\Gamma \propto k^{2}$ above. Related to the Ioffe-Regel crossover, this critical wave vector has been successively identified as the limit above which the spectra are mostly determined by the local order and similar to the ones of the polycrystal with the same local order and density. Here the peak acquires contributions from the transverse modes and from the higher-energy optic modes, not being then a single plane wave anymore [66]. We can thus expect our model not to work anymore in this regime.

Similarly to the case of glycerol, we report in Fig. 6 the boson peak, the velocity as modified by the presence of strings, and the excess attenuation due to the strings. The same considerations as above hold true for silica, both on the effect 


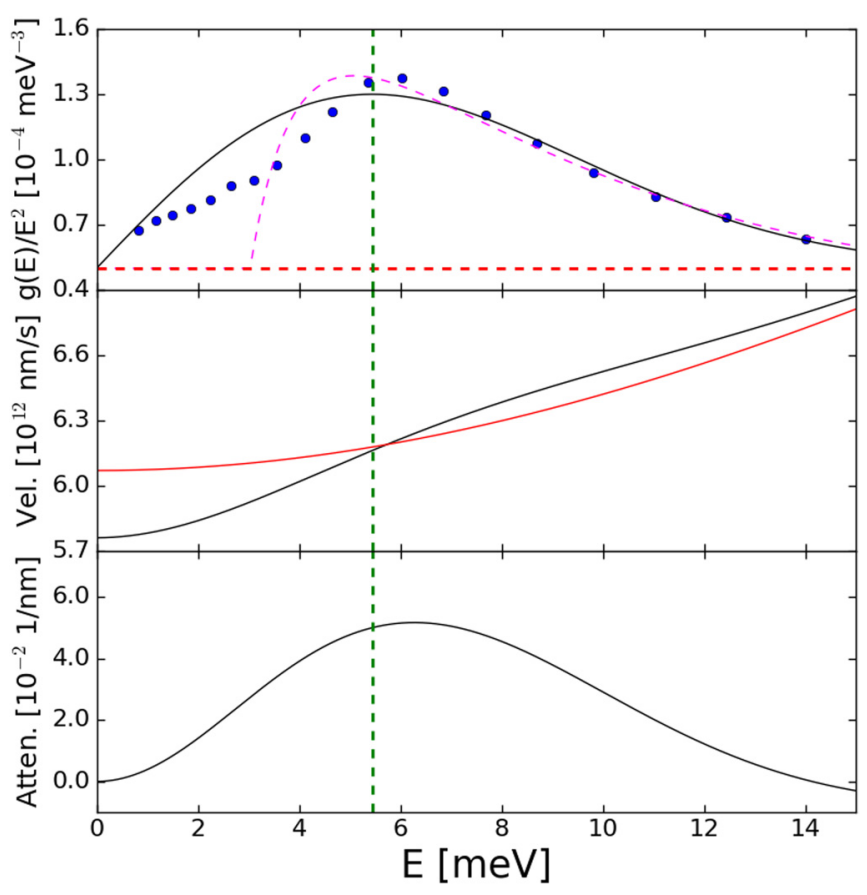

FIG. 6. Top panel: Excess of modes (boson peak) in the regular density of states of glassy silica and its modeling with the same function as in glycerol. The red dashed line represents what we should have in the absence of strings (no excess). Central panel: The velocity as obtained using Eq. (31), compared with the one in the case of no strings (red). Bottom panel: The excess attenuation, due to strings, with respect to the attenuation in the case of no strings. Its maximum, marked by the vertical green dashed line, corresponds to the change of behavior in the velocity from a lower to a higher velocity with respect to the no-string case, and to the maximum of the BP.

of strings and on the different crossover position with respect to the findings of the DHO model.

\section{DISCUSSION}

\section{A. The string distribution}

We have seen that our string model is able to provide a fit to experimental data as good as the one historically obtained with the DHO model. The derived velocity and attenuation, as calculated from the fitting parameters and Eqs. (31) and (32), can be clearly interpreted in terms of interference of the elastic waves with low-energy localized modes from strings with different lengths, giving a straightforward understanding of the elastic anomalies in glasses.

It is interesting to underline that in the examples above we have made a series of simplifications, which allows us to extract the important parameters of the model: (i) we have neglected the intrinsic damping in the strings, by imposing $B / m=0$; (ii) we have assumed $\alpha=1$, which corresponds to saying that the linear mass density of the string is the same as in the material; and (iii) we have taken the speed of sound $c$ in the string equal to the speed of shear waves: $c=c_{T}$. Moreover, the excess density of states is fixed by the experimental observation. The parameter $b$ (cross-sectional size of the string), still present in the model, cancels out, so

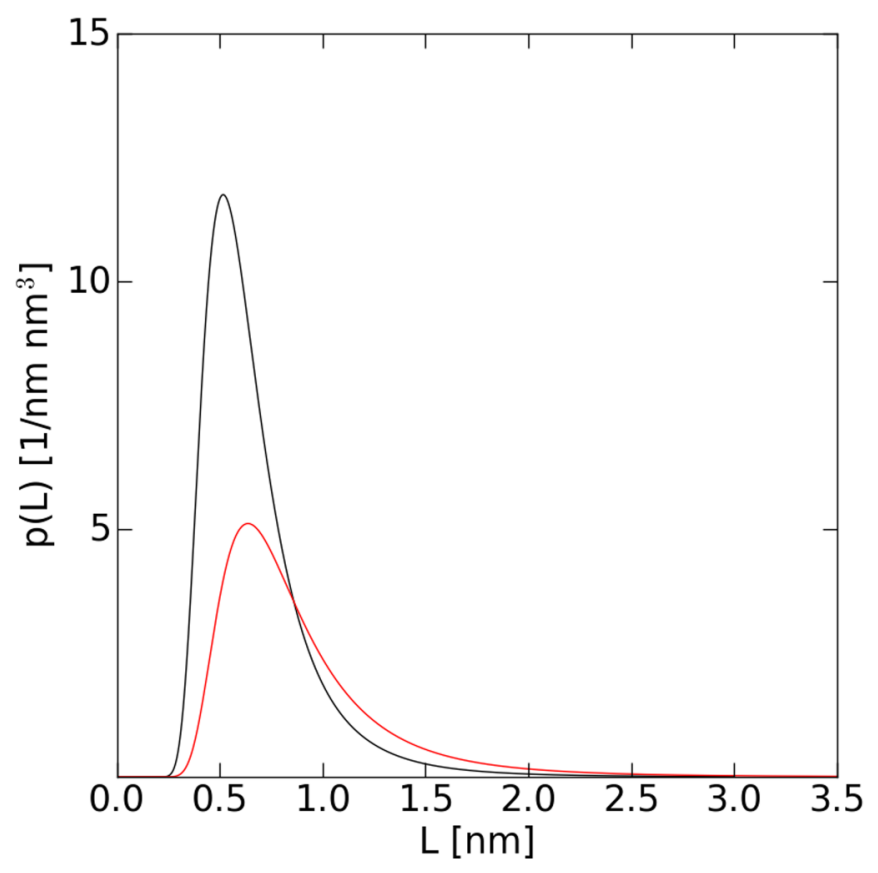

FIG. 7. We compare here the string length distribution as obtained from the boson peak in glassy glycerol (black) and in silica (red).

that the dynamics depends only on the strings' length, whose distribution leads to the distribution of string vibrational modes and then to the boson peak. As such, our fitting model is almost with no free parameters once the boson peak has been measured, which unambiguously fixes the string length distribution. The quality of the agreement of the model with the data is then impressive. In Fig. 7 we report the length distribution obtained for glycerol and silica.

Despite the large structural and dynamical differences between these two glasses, the boson peak is located at very similar energies, with a maximum, in reduced units, at $4 \mathrm{meV}$ in glycerol and $6 \mathrm{meV}$ in silica. This may explain the similarity of the string length distributions reported in the figure, with a slight difference in the position of the maximum, which well mimics the one in the BP position, the maximum being $0.52 \mathrm{~nm}$ and $0.64 \mathrm{~nm}$ for glycerol and silica, respectively. Such value corresponds well to the size of the elastic heterogeneities as found for silica in [66], and is also the typical size of the dynamical heterogeneities in glass-forming liquids close to the glass transition, suggesting a direct link between our strings and these latter [67,68]. It is also remarkable that in both glasses most of strings' lengths lie between about $0.4 \mathrm{~nm}$ and $2.6 \mathrm{~nm}$, implying that the same string modes are present in both glasses. It is worth noticing that $0.4 \mathrm{~nm}$ corresponds well to the correlation length for the spatial fluctuations of the local sound velocity, as identified in silica in [69]. Beyond these similarities, the two glasses' distributions differ for their integral area, which is directly connected to the boson peak amplitude, and corresponds to the total string number density: $5.012 \mathrm{~nm}^{-3}$ for glycerol and $3.177 \mathrm{~nm}^{-3}$ for silica $\left(3.129 \mathrm{~nm}^{-3}\right.$ for the 5-degree polynomial fit). 
In light of these observations, one may wonder whether the extent of the string distribution as well as a maximum around $0.5-0.6 \mathrm{~nm}$ are somehow universal parameters independent of the glass and of the position and intensity of the BP, which are only dependent on the finer details of the distribution (such as the precise position of the maximum and the total integral). Indeed, glycerol is a so-called intermediate glass, with fragility $m=50-53$ [70], against the strong character of silica, $m=19.8$ [71]. Fragility has been recently related to the microscopic connectivity [72], the fragility decreasing with the increasing of the network rigidity or connectivity. It would then be interesting to further investigate the relation between string length distribution and microscopic connectivity, by applying our model to a very fragile glass $(m \geqslant 90)$. More generally, applications of the model to more systems would help in understanding the role played by the length distribution detailed shape in determining the position of the excess of modes and shape (the BP), and its correlation with the fragility of the glass, and identify the universal features.

\section{B. Strings and low-energy localized modes from numerical simulations}

Very recently large numerical simulations have proved the existence in glasses of low-energy localized nonphononic modes at low energy. It is interesting to investigate further the link which can exist between the strings in our theory and such modes. These latter correspond to particle displacements decaying as $r^{-2}$ in 3D from the localization point [41-45], the same decay law being obeyed by the plastic events associated with quasistatic deformations. Interestingly, their density of states grows like frequency to the fourth in two, three, as well as four dimensions for energies below the boson peak [45]. In order to check whether the string vibrations present the same characteristics as the low-energy localized modes reported in these works, we calculate the particle displacement associated with them, solving Eqs. (A2) and (A3).

Consider a string segment of length $L$ along the $z$ axis, oscillating with a small amplitude $\delta$ and fundamental frequency $\omega_{0}$ along the $y$ direction. The displacement $X_{n}\left(s, t^{\prime}\right)$ as a function of a Lagrangian variable $s$ along the string and time $t^{\prime}$ is

$$
X_{n}\left(s, t^{\prime}\right)=\left(0, \delta e^{i \omega_{0} t^{\prime}} \sin \left[\frac{\pi}{L}\left(s+\frac{L}{2}\right)\right], s\right)
$$

with $-L / 2<s<L / 2$, equilibrium position $X_{n}^{0}(s)=$ $(0,0, s), b_{i}=(0,0,1)$ where $m=\rho b^{2}$, and $\tau_{j}=(0,0,1)$ is the tangent to the string (see Appendix A). In this case the particle velocity obtained from (A2) and (A3) is

$$
\begin{aligned}
v_{m}\left(\vec{x}, \omega_{0}\right) \\
=-i b \mu \omega_{0} \delta \int_{-L / 2}^{L / 2} d s \sin \left[\frac{\pi}{L}\left(s+\frac{L}{2}\right)\right] \\
\quad \times\left[\nabla_{1} G_{3 m}^{0}\left(\vec{x}-\vec{X}_{0}(s), \omega_{0}\right)+\nabla_{3} G_{1 m}^{0}\left(\vec{x}-\vec{X}_{0}(s), \omega_{0}\right)\right],
\end{aligned}
$$

where $G_{i j}^{0}$ is the bare Green's function introduced in Appendix A. For our present purposes we need it in the
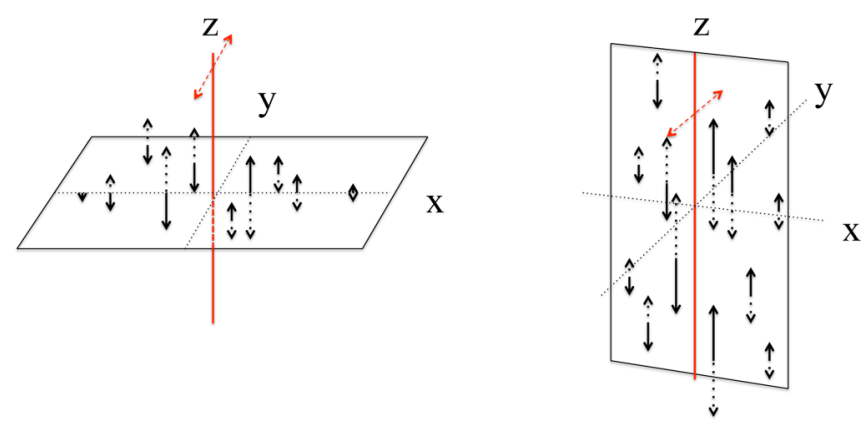

FIG. 8. Particle motion associated with string motion, according to Eqs. (33) and (36), for particles very close to the string $(r \ll L)$. The string (red straight line) points along the $z$ axis and moves perpendicular to itself, along the $y$ axis. Particles move along a direction parallel to the string, the $z$ axis. Left panel: Motion of particles lying along the $x-y$ plane. Right panel: Motion of particles along $x-z$. Here the particle motion is invariant with respect to translations along the $z$ axis. Note that the string and its (perpendicular) motion determine a plane, here the $y-z$ plane. Particle motion on one side of this plane, say positive $x$, is $180^{\circ}$ out of phase with respect to particle motion on the other side, negative $x$.

representation [73]

$$
\begin{aligned}
4 \pi \rho G_{k m}^{0}\left(\vec{r}, \omega_{0}\right) \\
=\frac{1}{c_{T}^{2} r} \delta_{k m} e^{-i \omega_{0} r / c_{T}}+\frac{r_{k} r_{m}}{r^{3}}\left[\frac{1}{c_{L}^{2}} e^{-i \omega_{0} r / c_{L}}-\frac{1}{c_{T}^{2}} e^{-i \omega_{0} r / c_{T}}\right] \\
\quad+\left(\frac{1}{r}\right)_{k m}\left[\frac{-1}{i \omega_{0}}\left(\frac{r}{c_{T}} e^{-i \omega_{0} r / c_{T}}-\frac{r}{c_{L}} e^{-i \omega_{0} r / c_{L}}\right)\right. \\
\left.\quad+\frac{1}{\omega_{0}^{2}}\left(e^{-i \omega_{0} r / c_{T}}-e^{-i \omega_{0} r / c_{L}}\right)\right] .
\end{aligned}
$$

After substitution of this expression into (34) we can identify three distinct regimes as a function of distance from the string:

(a) $r \ll L$ : that is, particle motion very close to the string, at distances small compared to string length. In this case it is straightforward to verify that, far from the pinning points, $v_{1}=v_{2}=0$ and that

$$
u_{3}\left(x, y ; \omega_{0}\right) \propto \frac{x}{x^{2}+y^{2}},
$$

where $(x, y)$ are coordinates on a plane centered on the string and perpendicular to it. This means that particles sharing the same $(x, y)$ coordinates will have the same motion, parallel to the string. This latter however moves perpendicular to itself, as it moves along the $y$ direction while it is aligned along the $z$ direction. As such, the particle displacement generated by the string corresponds to a transverse excitation, as can be better appreciated from Fig. 8. This motion seems compatible with that reported by Schober et al. $[49,74,75]$ in numerical simulations.

(b) $r \sim L$ : distances on the order of the string length. Remember that $\omega_{0} \sim c / L$. In this case, omitting terms depending on the string acceleration, which will be considered in (c), the particle displacement decays like $r^{-2}$ away from the center of the string, in a very nice agreement with the results of 
Lerner et al. [42-45] for the eigenvectors of the localized modes of a disordered solid.

(c) $r \gg L$ : distances very far from the string. In this case $v_{m}\left(r, \omega_{0}\right) \sim 1 / r$. This is the typical decay of a radiation field and was to be expected. Indeed, the vibrating string picture encapsulates the time variation of an inhomogeneous strain distribution. An inevitable result of such a time evolution is the generation of a radiating field that propagates away to infinity. This part of the particle displacement however emerges only after considering its time evolution; it depends on the string acceleration and cannot be obtained from a diagonalization of the dynamical matrix as calculated in Refs. [41-45].

So the string plays the role of a one-dimensional dynamical object that encapsulates the coherent motion of a localized, three-dimensional set of particles. More precisely, the boson peak normal modes correspond to motion of a localized set of particles in three-dimensional space, whose dynamics is described by a one-dimensional string of finite length.

Once we have assessed that the particle displacement related to a string motion is compatible with the one of the nonphonon low-energy localized modes, we next need to understand whether the density of states of the localized modes is also the same. The low-energy $\sim \omega^{4}$ behavior is actually not incompatible with the experimental density of states we have used in previous sections. In terms of strings, it would translate into a density of string lengths $\sim L^{-6}$ at large $L$.

Finally, the strings we have been considering are a specific implementation of nonaffine displacements. As such, they can account for both the excess number of vibrational states and the dispersive properties of acoustic waves, in glasses. In this sense, our work is intimately in agreement with the recent results by Caroli and Lemaitre, who have directly solved Newton's equation for a system of particles interacting through a soft-sphere potential in two dimensions, and highlighted the major role played by nonaffine displacements in the acoustic behavior of amorphous solids [20]. It would be interesting to extend the string picture to two-dimensional glasses to further relate this picture to the cited findings.

\section{CONCLUSION}

We have presented in this paper an implementation of the analytical string theory as developed by one of us for the description of the physics of dislocations, and later applied to the description of glasses, modeled as continuum media with embedded elastic string heterogeneities [52]. In that previous work it was already shown that such a description can account for the elastic anomalies reported at frequencies comparable with the boson peak: the strong phonon scattering and the negative dispersion in the sound velocity. Here we further develop that theory, in order to get the density-density correlation function and compare with experimental data obtained by inelastic x-ray scattering on two prototypical glasses, glycerol and silica.

We show that, once the vibrational density of states has been measured, we can use it for unambiguously fixing the string length distribution inherent to that glassy system. The density-density correlation function obtained using such dis- tribution is strongly constrained, and able to account for the experimental data in a fairly extended wave vector range, with the refinement of just a few fitting parameters. As such, the quality of the fit in the large wave vector range around the boson peak region is quite impressive for both systems.

From such fits, we can infer that the sound velocity and the attenuation of the elastic waves traveling in the continuum medium are perturbed due to the interference with the string vibrational eigenmodes: longer strings, characterized by lowenergy eigenmodes, slow down the sound wave, while shorter strings, whose eigenmodes correspond to the high-frequency tail of the boson peak, speed it up again. The maximum of the BP corresponds to a critical energy at which the change from negative to positive interference in the speed of sound takes place, as well as to the maximum of the extra attenuation, due to the interference with the strings. While the phenomenology closely resembles the one evidenced in [2], this critical energy is slightly smaller. However, as explained in Appendix B, it is not expected to obtain the same value.

We have shown that the string distribution model is compatible with the recent theoretical advances in the understanding of the vibrational properties of glasses in the sub-THz$\mathrm{THz}$ range. More specifically, the atomic motion associated with the strings presents a similar spatial amplitude behavior to the one obtained for the low-energy nonphononic modes in recent numerical simulations, allowing us to directly connect such nonphononic low-energy excess modes with the fundamental eigenmodes of the elastic heterogeneities represented by the strings.

It is thus interesting to critically inspect the string length distribution and its connection with the vibrational density of states and elastic anomalies. We have shown some common features, such as the range of string lengths and a peak at about $0.5-0.6 \mathrm{~nm}$, which are compatible with the reported elastic heterogeneities correlation length for silica [66] and dynamical heterogeneities in supercooled liquids $[67,68]$. Such common properties suggest a possible universality, beyond the fragility and the microscopic connectivity of the glass, which could be instead more closely related to the string density or the finer details of the distribution.

Our work thus not only offers a powerful tool, allowing us to directly obtain the longitudinal density-density correlation function from the density of states measurement, but also offers a microscopic understanding of the vibrational properties of glasses in terms of interference of the sound plane waves with low-energy modes of elastic 1D heterogeneities, compatible with the most recent theoretical findings. Such a picture allows us to identify the string length distribution features as key players in determining the vibrational anomalies, showing the universal character of some of them against the material-specific character of others.

Several future ways of investigation can be anticipated here. Our approach is expected to be universal, as it is based on continuum mechanics; thus its overall features should hold for all glasses, down to length scales of a few interatomic spacings. Still, the quantitative details should differ from glass to glass, depending on their specific microstructure. The identification of very similar string length distributions in two glasses with fairly different fragility requires further work for shedding light on this point, and identifying the universal or 
material-specific features of the string distribution. For this, the application of the model to a large number of systems with fragility spanning up to values as large as 90 and different microscopic connectivity is foreseen.

Second, the many experimental observations on the pressure and temperature dependence of the BP naturally raise the question about how the thermodynamic conditions impact the string distribution. It is clear that an effect will arise already for the induced density change; still the possible microstructure change should also reflect in modifications in the string distribution.

Finally, the reported success of our theory in describing the elastic anomalies in glasses, at energies in the $\mathrm{THz}$ range and nanometric wavelength, represents a solid basis for further developing the model for trying to understand other glassspecific dynamical phenomena. For example, an investigation of the string dynamics at temperatures lower than $1 \mathrm{~K}$, where quantum effects are likely to dominate, could shed light on the nature of two-level systems (TLS), or the implementation of the coupling of the strings with electromagnetic radiation would allow us to compare our model with a large amount of experimental data from optical spectroscopies (infrared, Raman, hyper-Raman, etc.), allowing us also to identify the limits of our description.

\section{ACKNOWLEDGMENTS}

This work has been supported by Fondecyt Grant No. 1191179 and ECOS-CONICYT Grant No. C17E02. The authors are grateful to G. Monaco for fruitful discussions and to G. Baldi for the $\mathrm{SiO}_{2}$ data sharing.

\section{APPENDIX A: COHERENT WAVES, STRINGS, AND THE BOSON PEAK [52,53,76]}

Consider a set of elastic strings of length $L$ with pinned end points. There are $p(L) d L$ strings with length between $L$ and $L+d L$ per unit volume. Each string is described by a displacement $\vec{X}(s, t)$ away from a straight equilibrium position $\vec{X}_{0}$, with $s$ a Lagrangian parameter along the string, which obeys the equation of an elastic string loaded by an external stress which, in the present context, will be associated with an elastic wave:

$$
m \ddot{X}_{k}(s, t)+B \dot{X}_{k}(s, t)-\mathcal{T} X_{k}^{\prime \prime}(s, t)=\mu b \mathrm{~N}_{k j p} \nabla_{j} u_{p}(\vec{X}, t),
$$

where $m \sim \rho b^{2}$ is a mass per unit length, $b$ is a length of order one interatomic distance, $\mathcal{T} \sim \mu b^{2}$ is a line tension, $B$ is a phenomenological damping coefficient, and $\nabla_{l} u_{k}\left(\vec{X}_{0}, t\right)$ is the gradient of the displacement $\vec{u}(\vec{x}, t)$ associated with the incoming wave, evaluated at the string equilibrium position. $\mathrm{N}_{k j p} \equiv \epsilon_{k j m} \tau_{m} \tau_{p}+\epsilon_{k p m} \tau_{m} \tau_{j}$, where $\tau_{j}$ is the unit tangent along the string equilibrium position. A prime means differentiation with respect to $s$. The right-hand side of (A1) is the Peach-Koehler force for screw dislocations in elastic continua. This coupling ensures that only the shear modulus, and not the bulk modulus, will, later on, become frequency dependent, a fact that has been observed in the numerical simulations of Maruzzo et al. [77].
On the other hand, an elastic wave traveling in the presence of such a string will obey the equation [78,79]

$$
\rho \ddot{v}_{i}(\vec{x}, t)-c_{i j k l} \nabla_{j} \nabla_{l} v_{k}(\vec{x}, t)=s_{i}(\vec{x}, t),
$$

with

$$
s_{i}(\vec{x}, t)=c_{i j k l} \epsilon_{m n k} b \int_{\mathcal{L}} d s \dot{X}_{m}(s, t) \tau_{n} \tau_{l} \nabla_{j} \delta\left(x-X_{0}\right),
$$

where $c_{i j k l}=\lambda \delta_{i j} \delta_{k l}+\mu\left(\delta_{i k} \delta_{j l}+\delta_{i l} \delta_{j k}\right)$.

So the picture is as follows: an elastic wave hits an elastic string; the string responds according to Eq. (A1); this response is plugged into the right-hand side of Eq. (A2) to obtain the scattered field generated by this response. Actually plugging the solution of (A1) into (A2) leads to, in the frequency domain,

$$
-\rho \omega^{2} v_{i}(\vec{x}, \omega)-c_{i j k l} \nabla_{j} \nabla_{l} v_{k}(\vec{x}, \omega)=V_{i k} v_{k}(\vec{x}, \omega),
$$

where

$$
V_{i k}=\left.\mathcal{A} \mathrm{N}_{m i j} \nabla_{j} \delta\left(\vec{x}-\vec{X}_{0}\right) \mathrm{N}_{m l k} \nabla_{l}\right|_{\vec{x}=\vec{X}_{0}},
$$

with

$$
\mathcal{A} \equiv \frac{8}{\pi^{2}} \frac{(\mu b)^{2}}{m} \frac{L}{\left[\omega^{2}-(\pi c / L)^{2}-i \omega B / m\right]}, \quad c^{2} \equiv \mathcal{T} / m .
$$

The next step is to think of the right-hand side of (A4) as consisting, not of a single string, but of many strings, randomly located and oriented, and with a distribution of lengths given by $p(L)$. In this case the problem is of a wave traveling in a random medium, or more precisely, in a medium filled with random scatterers. In any case, it is possible to find a coherent wave solution that is described by a complex index of refraction. This is achieved through the computation of an effective, average, Green's function.

Consider the full Green's function for the problem (A4):

$$
\begin{array}{r}
\rho \omega^{2} G_{i m}(\vec{x}, \omega)+c_{i j k l} \nabla_{j} \nabla_{l} G_{k m}(\vec{x}, \omega) \\
=-\sum_{\text {strings }} V_{i k} G_{k m}(\vec{x}, \omega)-\delta_{i m} \delta(\vec{x}) .
\end{array}
$$

To this end the average Green's function is written as ("Dyson's equation")

$$
\langle\langle G\rangle\rangle=\left[\left(G^{0}\right)^{-1}-\Sigma\right]^{-1},
$$

where $G^{0}$ is the Green's function of the medium without dislocations (i.e., "free" or "bare") [79-81], for an infinite, homogeneous, and isotropic medium, and the double brackets $\langle\langle\cdot\rangle\rangle$ denote an average over the random variables characterizing the disordered medium, not to be confused with the thermal average considered in the text. The mass operator $\Sigma$ can be computed from the interaction $V$ according to a well-defined, perturbative procedure. It turns out that, due to the pointlike nature of the interaction in (A7), the perturbation series can be summed and the result is

$$
\Sigma_{i j}=-\int \sigma_{0}(L) p(L) d L\left[\frac{1}{5}\left(\delta_{i j}-\hat{k}_{i} \hat{k}_{j}\right) k^{2}+\frac{4}{15} k_{i} k_{j}\right]
$$

with

$$
\sigma_{0} \equiv \frac{2 \mathcal{A}}{1+\mathcal{A} I}, \quad I \equiv-i \omega^{3} \frac{1}{5 \pi} \frac{1}{\rho c_{T}^{5}}
$$


Clearly, just like $G^{0}$, the mass operator $\Sigma$, and consequently the effective Green's function $\langle\langle G\rangle\rangle$, split into longitudinal and transverse parts:

$$
\left\langle\left\langle G_{i j}\right\rangle\right\rangle=\left\langle\left\langle G_{L}\right\rangle\right\rangle \hat{k}_{i} \hat{k}_{j}+\left\langle\left\langle G_{T}\right\rangle\right\rangle\left(\delta_{i j}-\hat{k}_{i} \hat{k}_{j}\right) .
$$

For the purposes of the present work we are only interested in the longitudinal portion and, from (A8), the result is

$$
\left\langle\left\langle G_{L}\right\rangle\right\rangle^{-1}=\rho\left(-\omega^{2}+c_{L}^{2} k^{2}\right)+k^{2} \frac{4}{15} \int \sigma_{0}(\omega, L) p(L) d L .
$$

Comparing (15) and (A12) we obtain

$$
f(\omega)=-\frac{4}{15} \frac{1}{\rho c_{L}^{2}} \int \sigma_{0}(\omega, L) p(L) d L,
$$

which relates a population of strings with a distribution of lengths and the index of refraction of Sec. II B.

Putting together (A6), (A10), and (A13) we have

$$
f(\omega)=-f_{0} \int \frac{L p(L) d L}{\omega^{2}-(\pi c / L)^{2}-i\left[\omega B / m+2 \omega^{3} /\left(\alpha \omega_{T}^{2}\right)\right]},
$$

where

$$
f_{0} \equiv \frac{1}{\alpha} \frac{8}{15} \frac{8}{\pi^{2}} \frac{c_{T}^{4}}{c_{L}^{2}}, \quad \omega_{T}^{2} \equiv \frac{5 \pi^{3}}{4} \frac{c_{T}}{L},
$$

and $\alpha$ is a dimensionless parameter of order one defined by $m \equiv \alpha \rho b^{2}$.

\section{APPENDIX B: THE DAMPED HARMONIC OSCILLATOR MODEL}

We follow the book of Boon and Yip [56]; see also Scopigno et al. [57]. Starting from a linear description of a Navier-Stokes fluid with constant transport coefficient one finds, neglecting temperature fluctuations, that the current $j(\vec{x}, t)$ obeys the same Eq. (1) that has been our starting point for waves in an elastic solid, and, unsurprisingly, the current correlation is given by (11). That is, Navier-Stokes sound in a fluid and elastic sound in a continuous, homogeneous solid obey the same equations at low frequency and wave number.

In generalized hydrodynamics, the next step is to introduce a memory function into the equation for the current correlation function. The Navier-Stokes expression

$$
\frac{\partial J_{\mathrm{NS}}(k, t)}{\partial t}=-k^{2} c_{L}^{2} \int_{0}^{t} d t^{\prime} J_{\mathrm{NS}}(k, t)-v k^{2} J_{\mathrm{NS}}(k, t),
$$

where $v$ is the viscosity, is replaced by

$$
\begin{aligned}
\frac{\partial J_{\mathrm{GH}}(k, t)}{\partial t}= & -\frac{\left(k v_{0}\right)^{2}}{S(k)} \int_{0}^{t} d t^{\prime} J_{\mathrm{GH}}(k, t) \\
& -k^{2} \int_{0}^{t} d t^{\prime} \Phi\left(k, t-t^{\prime}\right) J_{\mathrm{GH}}(k, t) .
\end{aligned}
$$

Here, $v_{0}$ is the thermal particle velocity and $S(k)$ is the static structure factor. These quantities are determined by the material at hand, and the replacement of $c_{L}$ by $v_{0} / S(k)$ is dictated by one of the sum rules that must be obeyed by the current correlation function $J(k, t)$. The "memory function" $\Phi(k, t)$ is, as yet, undetermined, and is supposed to capture the fact that, at high frequencies and short wavelengths, viscous effects are no longer described by constant coefficients, but depend on time and length scales. The current correlation obtained from this expression is

$$
\begin{aligned}
& J_{\mathrm{GH}}(k, \omega) \\
& \quad=\frac{2 v_{0}^{2} \omega^{2} k^{2} \Phi^{\prime}(k, \omega)}{\left\{\omega^{2}-\left[\left(k v_{0}\right)^{2} / S(k)\right]+\omega k^{2} \Phi^{\prime \prime}(k, \omega)\right\}^{2}+\left[\omega k^{2} \Phi^{\prime}(k, \omega)\right]^{2}},
\end{aligned}
$$

where $\Phi^{\prime}$ and $\Phi^{\prime \prime}$ are the real and imaginary parts of the Laplace-Fourier transform of $\Phi(k, t)$.

The damped harmonic oscillator (DHO) expression employed in Refs. [2,17] to interpret the glycerol and silica data is obtained from (B3) with the special choices

$$
\Phi^{\prime \prime}(k, \omega)=0, \quad k^{2} \Phi^{\prime}(k, \omega) \equiv 2 \Gamma(k), \quad \frac{\left(k v_{0}\right)^{2}}{S(k)} \equiv \Omega^{2}(k) .
$$

That is, the damping is instantaneous (i.e., independent of frequency) but wave number dependent. The functions $\Gamma(k)$ and $\Omega(k)$ are obtained through a comparison with the experimental data, and they do not appear to be directly related to a specific particle behavior in the glass. They do reproduce, however, the Navier-Stokes behavior at long wavelengths.

In the body of this paper we have used an expression for the current correlation given by Eqs. (24), (15), and (A14). This expression differs from a DHO model. It can, however, be obtained as a special case of the generalized hydrodynamics expression, Eq. (B3), with

$$
\begin{gathered}
\omega \Phi^{\prime}=c_{L}^{2}(\xi \omega+\operatorname{Im} f), \\
\frac{v_{0}^{2}}{S(k)}-\omega \Phi^{\prime \prime}=c_{L}^{2}(1-\operatorname{Re} f) .
\end{gathered}
$$

These two formulas show that the string model developed in the body of the paper can be considered as being encompassed by a memory function formalism, albeit with a somewhat roundabout formulation. The coherent-wave picture developed in Sec. II provides a more direct formulation, that in addition has a straightforward physical interpretation, and it links the wave behavior to the vibrational degrees of freedom contained in the boson peak, through the function $f(\omega)$. These coherent waves are characterized by a phase velocity and attenuation given by Eqs. (31) and (32), which differ from the position divided by wave vector and full width at half maximum of the inelastic peak in the dynamic structure function, which are the definitions of velocity and attenuation commonly associated with the DHO model. Both the string and the DHO models provide adequate fits of the experimental data. They differ, however, in terms of physical interpretation.
[1] C. Kittel, Introduction to Solid State Physics, 8th ed. (John Wiley and Sons, 2005).
[2] G. Monaco and V. M. Giordano, Proc. Natl. Acad. Sci. USA 106, 3659 (2009). 
[3] U. Buchenau, N. Nücker, and A. J. Dianoux, Phys. Rev. Lett. 53, 2316 (1984).

[4] R. C. Zeller and R. O. Pohl, Phys. Rev. B 4, 2029 (1971).

[5] E. Courtens, M. Foret, B. Hehlen, and R. Vacher, Solid State Commun. 117, 187 (2001).

[6] A. Wischnewski, U. Buchenau, A. J. Dianoux, W. A. Kamitakahara, and J. L. Zarestky, Phys. Rev. B 57, 2663 (1998).

[7] S. Caponi, A. Fontana, F. Rossi, G. Baldi, and E. Fabiani, Phys. Rev. B 76, 092201 (2007).

[8] Y. Inamura, M. Arai, T. Otomo, N. Kitamura, and U. Buchenau, Phys. B (Amsterdam) 284-288, 1157 (2000).

[9] M. Zanatta, G. Baldi, S. Caponi, A. Fontana, E. Gilioli, M. Krish, C. Masciovecchio, G. Monaco, L. Orsingher, F. Rossi, G. Ruocco, and R. Verbeni, Phys. Rev. B 81, 212201 (2010).

[10] A. Monaco, A. I. Chumakov, G. Monaco, W. A. Crichton, A. Meyer, L. Comez, D. Fioretto, J. Korecki, and R. Rüffer, Phys. Rev. Lett. 97, 135501 (2006).

[11] L. Orsingher, A. Fontana, E. Gilioli, G. Carini Jr., G. Carini, G. Tripodo, T. Unruh, and U. Buchenau, J. Chem. Phys. 132, 124508 (2010).

[12] K. Niss, B. Begen, B. Frick, J. Ollivier, A. Beraud, A. Sokolov, V. N. Novikov, and C. Alba-Simionesco, Phys. Rev. Lett. 99, 055502 (2007).

[13] L. Hong, B. Begen, A. Kisliuk, C. Alba-Simionesco, V. N. Novikov, and A. P. Sokolov, Phys. Rev. B 78, 134201 (2008).

[14] S. Caponi, S. Corezzi, D. Fioretto, A. Fontana, G. Monaco, and F. Rossi, Phys. Rev. Lett. 102, 027402 (2009).

[15] A. Monaco, A. I. Chumakov, Y.-Z. Yue, G. Monaco, L. Comez, D. Fioretto, W. A. Crichton, and R. Rüffer, Phys. Rev. Lett. 96, 205502 (2006).

[16] G. Baldi, V. M. Giordano, G. Monaco, and B. Ruta, Phys. Rev. Lett. 104, 195501 (2010).

[17] G. Baldi, V. M. Giordano, and G. Monaco, Phys. Rev. B 83, 174203 (2011).

[18] B. Ruta, G. Baldi, F. Scarponi, D. Fioretto, V. M. Giordano, and G. Monaco, J. Chem. Phys. 137, 214502 (2012).

[19] S. Gelin, H. Tanaka, and A. Lemaître, Nat. Mater. 15, 1177 (2016).

[20] C. Caroli and A. Lemaître, Phys. Rev. Lett. 123, 055501 (2019).

[21] Y. M. Beltukov, C. Fusco, D. A. Parshin, and A. Tanguy, Phys. Rev. E 93, 023006 (2016).

[22] A. Tanguy, J. P. Wittmer, F. Leonforte, and J.-L. Barrat, Phys. Rev. B 66, 174205 (2002).

[23] P. B. Allen, J. L. Feldman, J. Fabian, and F. Wooten, Philos. Mag. B 79, 1715 (1999).

[24] P. B. Allen and J. L. Feldman, Phys. Rev. B 48, 12581 (1993).

[25] J. J. Ludlam, S. N. Taraskin, and S. R. Elliott, Phys. Rev. B 67, 132203 (2003).

[26] D. R. Parshin, Fiz. Tverd. Tela (Leningrad) 36, 1809 (1994); Phys. Solid State 36, 991 (1994).

[27] W. Götze and M. R. Mayr, Phys. Rev. E 61, 587 (2000).

[28] W. Schirmacher, G. Diezemann, and C. Ganter, Phys. Rev. Lett. 81, 136 (1998).

[29] W. Schirmacher, Europhys. Lett. 73, 892 (2006).

[30] W. Schirmacher, G. Ruocco, and T. Scopigno, Phys. Rev. Lett. 98, 025501 (2007).

[31] T. S. Grigera, V. Martín-Mayor, G. Parisi, and P. Verrocchio, Nature (London) 422, 289 (2003).

[32] C. Ganter and W. Schirmacher, Phys. Rev. B 82, 094205 (2010).
[33] Y. M. Beltukov, V. I. Kozub, and D. A. Parshin, Phys. Rev. B 87, 134203 (2013).

[34] U. Buchenau, Y. M. Galperin, V. L. Gurevich, and H. R. Schober, Phys. Rev. B 43, 5039 (1991).

[35] V. L. Gurevich and H. R. Schober, Phys. Rev. B 57, 11295 (1998).

[36] V. L. Gurevich, D. A. Parshin, and H. R. Schober, Phys. Rev. B 67, 094203 (2003).

[37] E. Duval, A. Mermet, and L. Saviot, Phys. Rev. B 75, 024201 (2007).

[38] J. W. Kantelhardt, S. Russ, and A. Bunde, Phys. Rev. B 63, 064302 (2001).

[39] S. N. Taraskin, Y. L. Loh, G. Natarajan, and S. R. Elliott, Phys. Rev. Lett. 86, 1255 (2001).

[40] A. I. Chumakov, G. Monaco, A. Monaco, W. A. Crichton, A. Bosak, R. Rüffer, A. Meyer, F. Kargl, L. Comez, D. Fioretto, H. Giefers, S. Roitsch, G. Wortmann, M. H. Manghnani, A. Hushur, Q. Williams, J. Balogh, K. Parliński, P. Jochym, and P. Piekarz, Phys. Rev. Lett. 106, 225501 (2011).

[41] M. Shimada, H. Mizuno, and A. Ikeda, Phys. Rev. E 97, 022609 (2018).

[42] E. Lerner, G. Düring, and E. Bouchbinder, Phys. Rev. Lett. 117, 035501 (2016).

[43] L. Gartner and E. Lerner, Phys. Rev. E 93, 011001(R) (2016).

[44] G. Kapteijns, E. Bouchbinder, and E. Lerner, Phys. Rev. Lett. 121, 055501 (2018).

[45] E. Lerner and E. Bouchbinder, J. Chem. Phys. 148, 214502 (2018).

[46] C. Donati, J. F. Douglas, W. Kob, S. J. Plimpton, P. H. Poole, and S. C. Glotzer, Phys. Rev. Lett. 80, 2338 (1998).

[47] H. Zhang, C. Zhong, J. F. Douglas, X. Wang, Q. Cao, D. Zhang, and J.-Z. Jiang, J. Chem. Phys. 142, 164506 (2015).

[48] H. B. Yu, K. Samwer, W. H. Wang, and H. Y. Bai, Nat. Commun. 4, 2204 (2013).

[49] H. Schober, C. Oligschleger, and B. Laird, J. Non-Cryst. Solids 156-158, 965 (1993).

[50] V. N. Novikov and N. V. Surovtsev, Phys. Rev. B 59, 38 (1999).

[51] A. Concustell, S. Godard-Desmarest, M. Carpenter, N. Nishiyama, and A. Greer, Scr. Mater. 64, 1091 (2011).

[52] F. Lund, Phys. Rev. B 91, 094102 (2015).

[53] D. Churochkin, F. Barra, F. Lund, A. Maurel, and V. Pagneux, Wave Motion 60, 220 (2016).

[54] L. D. Landau and E. M. Lifshitz, Theory of Elasticity, 2nd ed. (Pergamon Press, 1989).

[55] P. M. Morse and H. Feshbach, Methods of Theoretical Physics (McGraw Hill, 1953).

[56] J. P. Boon and S. Yip, Molecular Hydrodynamics (Dover Publications, 2013).

[57] T. Scopigno, G. Ruocco, and F. Sette, Rev. Mod. Phys. 77, 881 (2005).

[58] S. Pailhès, V. M. Giordano, P.-F. Lory, M. De Boissieu, and H. Euchner, in Amorphisation and Thermal Properties of Semiconductors: From Bulk to Nanostructures, edited by K. Termentzidis (Pan Stanford, CRC Press/Taylor \& Francis Group, 2017), Chap. 19.

[59] F. Lund, J. Mater. Res. 3, 280 (1988).

[60] J. Wuttke, W. Petry, G. Coddens, and F. Fujara, Phys. Rev. E 52, 4026 (1995). 
[61] L. Comez, D. Fioretto, F. Scarponi, and G. Monaco, J. Chem. Phys. 119, 6032 (2003).

[62] F. Scarponi, L. Comez, D. Fioretto, and L. Palmieri, Phys. Rev. B 70, 054203 (2004).

[63] A. G. Lyapin, E. Gromnitskaya, I. V. Danilov, and V. V. Brazhkin, RSC Adv. 7, 33278 (2017).

[64] W. Pabst and E. Gregorová, Ceramics-Silikaty 57, 167 (2013).

[65] G. Baldi, V. M. Giordano, G. Monaco, F. Sette, E. Fabiani, A. Fontana, and G. Ruocco, Phys. Rev. B 77, 214309 (2008).

[66] G. Baldi, M. Zanatta, E. Gilioli, V. Milman, K. Refson, B. Wehinger, B. Winkler, A. Fontana, and G. Monaco, Phys. Rev. Lett. 110, 185503 (2013).

[67] U. Tracht, M. Wilhelm, A. Heuer, H. Feng, K. Schmidt-Rohr, and H. W. Spiess, Phys. Rev. Lett. 81, 2727 (1998).

[68] L. Berthier, G. Biroli, J.-P. Bouchaud, L. Cipelletti, D. E. Masri, D. L'Hôte, F. Ladieu, and M. Pierno, Science 310, 1797 (2005).

[69] G. Baldi, V. M. Giordano, B. Ruta, R. Dal Maschio, A. Fontana, and G. Monaco, Phys. Rev. Lett. 112, 125502 (2014).

[70] M. Nakanishi and R. Nozaki, Phys. Rev. E 83, 051503 (2011).
[71] M. L. F. Nascimento and C. Aparicio, J. Phys. Chem. Solids 68, 104 (2007).

[72] D. L. Sidebottom, Front. Mater. 6, 144 (2019).

[73] A. E. H. Love, A Treatise on the Mathematical Theory of Elasticity (Wentworth Press, 2019).

[74] H. R. Schober, C. Gaukel, and C. Oligschleger, Prog. Theor. Phys. Suppl. 126, 67 (1997).

[75] H. Schober, J. Non-Cryst. Solids 307-310, 40 (2002).

[76] N. Mujica, M. T. Cerda, R. Espinoza, J. Lisoni, and F. Lund, Acta Mater. 60, 5828 (2012).

[77] A. Marruzzo, W. Schirmacher, A. Fratalocchi, and G. Ruocco, Sci. Rep. 3, 1407 (2013).

[78] A. Maurel, V. Pagneux, F. Barra, and F. Lund, Phys. Rev. B 72, 174110 (2005).

[79] A. Maurel, V. Pagneux, F. Barra, and F. Lund, Phys. Rev. B 72, 174111 (2005).

[80] R. Weaver, J. Mech. Phys. Solids 38, 55 (1990).

[81] A. Maurel, J.-F. Mercier, and F. Lund, Phys. Rev. B 70, 024303 (2004). 